\title{
Power-Expected-Posterior Priors for Generalized Linear Models
}

by D.Fouskakis, I.Ntzoufras and K.Perrakis

\section{Electronic Appendix}

\section{Appendix A: Proofs of Predictive Matching}

\section{A.1 Proof of Proposition 1.}

Assuming known and common across all models $\phi_{\gamma}=\phi$, then, for samples of size $n=1$ (and, therefore we have also $\delta=n^{*}=1$ ), both CR-PEP and DR-PEP priors coincide. Moreover, assuming that we have observed the response $y$ with covariate values $\boldsymbol{x}=\left(x_{1}, \ldots, x_{p}\right)^{T}$ then we need to generate an imaginary data-point $y^{*}$ under the same set of covariates. Moreover, the linear predictor for any model $\gamma$ is now given by

$$
\eta_{\boldsymbol{\gamma}}=\beta_{0, \boldsymbol{\gamma}}+\boldsymbol{x}_{\boldsymbol{\gamma}}^{T} \boldsymbol{\beta}_{\backslash 0, \boldsymbol{\gamma}}=\beta_{0, \boldsymbol{\gamma}}+\sum_{j=1}^{p} \gamma_{j} x_{j} \beta_{j}
$$

where $\boldsymbol{x}_{\boldsymbol{\gamma}}$ is the sub-vector of $\boldsymbol{x}$ with elements corresponding to covariates included in model $M_{\gamma}$.

Under this formulation and a prior as defined in Proposition 1, the prior predictive density for model $M_{0}$ under the baseline prior $\pi_{0}^{N}\left(\beta_{0}\right)$ is given by

$$
m_{0}^{\mathrm{N}}\left(y^{*} \mid \delta=1\right)=\int \exp \left(\frac{y^{*} \vartheta\left(\beta_{0}\right)-b\left(\vartheta\left(\beta_{0}\right)\right)}{a(\phi)}+c\left(y^{*}, \phi\right)\right) \psi\left(\beta_{0}\right) d \beta_{0} .
$$

By setting $\eta=\beta_{0}$ under model $M_{0}$, we obtain

$$
\begin{aligned}
m_{0}^{\mathrm{N}}\left(y^{*} \mid \delta=1\right)= & e^{c\left(y^{*}, \phi\right)} \mathcal{D}\left(y^{*}, 1,1\right) \\
& \text { where } \mathcal{D}(y, \omega, \delta)=\int \exp \left(\frac{y^{*} \vartheta(\eta)-\omega b(\vartheta(\eta))}{\delta a(\phi)}\right) \psi(\eta) d \eta .
\end{aligned}
$$

For model $M_{\boldsymbol{\gamma}}$, the prior predictive density under the baseline prior $\pi_{\gamma}^{N}\left(\boldsymbol{\beta}_{\gamma}\right)$ is given by

$$
m_{\boldsymbol{\gamma}}^{\mathrm{N}}\left(y^{*} \mid \delta=1\right)=\int \exp \left(\frac{y^{*} \vartheta\left(\eta_{\boldsymbol{\gamma}}\right)-b\left(\vartheta\left(\eta_{\boldsymbol{\gamma}}\right)\right)}{a(\phi)}+c\left(y^{*}, \phi\right)\right) \psi\left(\eta_{\gamma}\right) \Psi_{\boldsymbol{\gamma}}\left(\boldsymbol{\beta}_{\backslash 0, \boldsymbol{\gamma}}\right) d \boldsymbol{\beta}_{\boldsymbol{\gamma}}
$$

By setting $\eta=\beta_{0, \boldsymbol{\gamma}}+\sum_{j=1}^{p} \gamma_{j} x_{j} \beta_{j}=\eta_{\boldsymbol{\gamma}}$ (see Eq. A.1) and $\boldsymbol{b}=\boldsymbol{\beta}_{\backslash 0, \boldsymbol{\gamma}}$, then from (A.3) we obtain

$$
m_{\gamma}^{\mathrm{N}}\left(y^{*} \mid \delta=1\right)=e^{c\left(y^{*}, \phi\right)} \int\left\{\int \exp \left(\frac{y^{*} \vartheta(\eta)-b(\vartheta(\eta))}{a(\phi)}\right) \psi(\eta) d \eta\right\} \Psi_{\boldsymbol{\gamma}}(\boldsymbol{b}) d \boldsymbol{b}
$$




$$
=e^{c\left(y^{*}, \phi\right)} \mathcal{D}\left(y^{*}, 1,1\right) \mathcal{A}_{\boldsymbol{\gamma}}=m_{0}^{N}\left(y^{*} \mid \delta=1\right) \mathcal{A}_{\boldsymbol{\gamma}},
$$

where $\mathcal{A}_{\boldsymbol{\gamma}}=\int \Psi_{\boldsymbol{\gamma}}(\boldsymbol{b}) d \boldsymbol{b}$.

The marginal likelihood of $M_{0}$ under the PEP prior is given by

$$
m_{0}^{\mathrm{PEP}}(y \mid \delta=1)=m_{0}^{N}(y \mid \delta=1),
$$

while for model $M_{\gamma}$ is given by

$$
\begin{aligned}
m_{\boldsymbol{\gamma}}^{\mathrm{PEP}}(y \mid \delta=1) & =\int \frac{m_{0}^{\mathrm{N}}\left(y^{*} \mid \delta=1\right)}{m_{\boldsymbol{\gamma}}^{\mathrm{N}}\left(y^{*} \mid \delta=1\right)}\left\{\int f_{\boldsymbol{\gamma}}\left(y \mid \boldsymbol{\beta}_{\boldsymbol{\gamma}}\right) f_{\boldsymbol{\gamma}}\left(y^{*} \mid \boldsymbol{\beta}_{\boldsymbol{\gamma}}, \delta=1\right) \pi_{\boldsymbol{\gamma}}^{\mathrm{N}}\left(\boldsymbol{\beta}_{\boldsymbol{\gamma}}\right) \mathrm{d} \boldsymbol{\beta}_{\boldsymbol{\gamma}}\right\} \mathrm{d} y^{*} \\
& =\int \mathcal{A}_{\boldsymbol{\gamma}}^{-1}\left\{\int f_{\boldsymbol{\gamma}}\left(y \mid \boldsymbol{\beta}_{\boldsymbol{\gamma}}\right) f_{\boldsymbol{\gamma}}\left(y^{*} \mid \boldsymbol{\beta}_{\boldsymbol{\gamma}}, \delta=1\right) \pi_{\boldsymbol{\gamma}}^{\mathrm{N}}\left(\boldsymbol{\beta}_{\boldsymbol{\gamma}}\right) \mathrm{d} \boldsymbol{\beta}_{\boldsymbol{\gamma}}\right\} \mathrm{d} y^{*} \\
& =\int \mathcal{A}_{\boldsymbol{\gamma}}^{-1}\left\{f_{\boldsymbol{\gamma}}\left(y \mid \boldsymbol{\beta}_{\boldsymbol{\gamma}}\right)\left[\int f_{\boldsymbol{\gamma}}\left(y^{*} \mid \boldsymbol{\beta}_{\boldsymbol{\gamma}}, \delta=1\right) \mathrm{d} y^{*}\right] \pi_{\boldsymbol{\gamma}}^{\mathrm{N}}\left(\boldsymbol{\beta}_{\boldsymbol{\gamma}}\right)\right\} \mathrm{d} \boldsymbol{\beta}_{\boldsymbol{\gamma}} \\
& =\mathcal{A}_{\boldsymbol{\gamma}}^{-1} \int f_{\boldsymbol{\gamma}}\left(y \mid \boldsymbol{\beta}_{\boldsymbol{\gamma}}\right) \pi_{\boldsymbol{\gamma}}^{\mathrm{N}}\left(\boldsymbol{\beta}_{\boldsymbol{\gamma}}\right) \mathrm{d} \boldsymbol{\beta}_{\boldsymbol{\gamma}}=\mathcal{A}_{\boldsymbol{\gamma}}^{-1} m_{\boldsymbol{\gamma}}^{N}(y \mid \delta=1) \\
& =\mathcal{A}_{\boldsymbol{\gamma}}^{-1} m_{0}^{N}(y \mid \delta=1) \mathcal{A}_{\boldsymbol{\gamma}}=m_{0}^{N}(y \mid \delta=1)
\end{aligned}
$$

and hence, for known $\phi$, this concludes the proof.

If $\phi$ is stochastic and common across models, then the two marginal likelihoods, obtained by integrating out $\phi$ over the common prior $\pi_{0}^{N}(\phi)=\pi_{\gamma}^{N}(\phi)$ for all models $M_{\gamma}$, still coincide.

\section{A.2 Proof of Proposition 2.}

Following similar arguments as in Section A.1 we have that

$$
\begin{aligned}
& m_{0}^{\mathrm{N}}\left(y^{*} \mid \delta\right)=e^{c\left(y^{*}, \phi\right) / \delta} \mathcal{D}\left(y^{*}, 1, \delta\right) \\
& m_{\gamma}^{\mathrm{N}}\left(y^{*} \mid \delta\right)=e^{c\left(y^{*}, \phi\right) / \delta} \mathcal{D}\left(y^{*}, 1, \delta\right) \mathcal{A}_{\boldsymbol{\gamma}}=m_{0}^{N}\left(y^{*} \mid \delta\right) \mathcal{A}_{\boldsymbol{\gamma}} .
\end{aligned}
$$

The final marginal likelihood of $M_{\gamma}$ under the DR-PEP prior, conditional on $\delta$, is given by

$$
\begin{aligned}
m_{\boldsymbol{\gamma}}^{\mathrm{DR}-\mathrm{PEP}}(y \mid \delta)= & \mathcal{C}_{0}^{-1} \int \frac{m_{0}^{\mathrm{N}}\left(y^{*} \mid \delta\right)}{m_{\boldsymbol{\gamma}}^{\mathrm{N}}\left(y^{*} \mid \delta\right)}\left\{\int f_{\boldsymbol{\gamma}}\left(y \mid \boldsymbol{\beta}_{\gamma}\right) f_{\boldsymbol{\gamma}}\left(y^{*} \mid \boldsymbol{\beta}_{\boldsymbol{\gamma}}, \delta\right) \pi_{\boldsymbol{\gamma}}^{\mathrm{N}}\left(\boldsymbol{\beta}_{\boldsymbol{\gamma}}\right) \mathrm{d} \boldsymbol{\beta}_{\boldsymbol{\gamma}}\right\} \mathrm{d} y^{*} \\
= & \mathcal{C}_{0}^{-1} \int \mathcal{A}_{\boldsymbol{\gamma}}^{-1}\left\{\int f_{\boldsymbol{\gamma}}\left(y \mid \boldsymbol{\beta}_{\boldsymbol{\gamma}}\right) f_{\boldsymbol{\gamma}}\left(y^{*} \mid \boldsymbol{\beta}_{\boldsymbol{\gamma}}, \delta\right) \pi_{\boldsymbol{\gamma}}^{\mathrm{N}}\left(\boldsymbol{\beta}_{\boldsymbol{\gamma}}\right) \mathrm{d} \boldsymbol{\beta}_{\boldsymbol{\gamma}}\right\} \mathrm{d} y^{*} \\
= & \mathcal{C}_{0}^{-1} \mathcal{A}_{\boldsymbol{\gamma}}^{-1} \iint \exp \left(\frac{y \vartheta\left(\eta_{\boldsymbol{\gamma}}\right)-b\left(\vartheta\left(\eta_{\boldsymbol{\gamma}}\right)\right)}{a(\phi)}+c(y, \phi)\right) \\
& \quad \times \exp \left(\frac{y^{*} \vartheta\left(\eta_{\boldsymbol{\gamma}}\right)-b\left(\vartheta\left(\eta_{\boldsymbol{\gamma}}\right)\right)}{\delta a(\phi)}+\frac{c\left(y^{*}, \phi\right)}{\delta}\right) \pi_{\boldsymbol{\gamma}}^{\mathrm{N}}\left(\boldsymbol{\beta}_{\boldsymbol{\gamma}}\right) \mathrm{d} \boldsymbol{\beta}_{\boldsymbol{\gamma}} \mathrm{d} y^{*} .
\end{aligned}
$$

Using a prior as defined in Proposition 1 and setting $\eta=\beta_{0, \gamma}+\sum_{j=1}^{p} \gamma_{j} x_{j} \beta_{j}=\eta_{\gamma}$ (see Eq. A.1) and $\boldsymbol{b}=\boldsymbol{\beta}_{\backslash 0, \gamma}$, we obtain

$$
m_{\gamma}^{\mathrm{DR}-\mathrm{PEP}}(y \mid \delta)=\mathcal{C}_{0}^{-1} \mathcal{A}_{\gamma}^{-1} \times
$$




$$
\begin{aligned}
& \times \int e^{c(y, \phi)+c\left(y^{*}, \phi\right) / \delta}\left\{\int \exp \left(\frac{\left(\delta y+y^{*}\right) \vartheta(\eta)-(\delta+1) b(\vartheta(\eta))}{\delta a(\phi)}\right) \psi(\eta) \mathrm{d} \eta \int \Psi_{\gamma}(\boldsymbol{b}) \mathrm{d} \boldsymbol{b}\right\} \mathrm{d} y^{*} . \\
= & \mathcal{C}_{0}^{-1} e^{c(y, \phi)} \int e^{c\left(y^{*}, \phi\right) / \delta} \mathcal{D}\left(\delta y+y^{*}, \delta+1, \delta\right) \mathrm{d} y^{*}
\end{aligned}
$$

which does not depend on the original model formulation $M_{\gamma}$. Indeed, following similar logic we can prove that

$$
m_{0}^{\mathrm{DR}-\mathrm{PEP}}(y \mid \delta)=\mathcal{C}_{0}^{-1} e^{c(y, \phi)} \int e^{c\left(y^{*}, \phi\right) / \delta} \mathcal{D}\left(\delta y+y^{*}, \delta+1, \delta\right) \mathrm{d} y^{*},
$$

and hence, for known $\phi$, it is obvious that after integrating out $\delta$ over the hyper-prior $\pi(\delta)$, $m_{\boldsymbol{\gamma}}^{\mathrm{DR}-\mathrm{PEP}}(y)=m_{0}^{\mathrm{DR}-\mathrm{PEP}}(y)$ and this concludes the proof.

If $\phi$ is stochastic and common across models, then the two marginal likelihoods, obtained by integrating out $\phi$ over the common prior $\pi_{0}^{N}(\phi)=\pi_{\gamma}^{N}(\phi)$ for all models $M_{\gamma}$, still coincide.

\section{A.3 Proof of Proposition 3.}

For the CR-PEP prior, conditional on $\delta$, using the prior in Proposition 1, (A.2) and (A.4) and following similar steps as in Appendix A.1 we have that

$$
\begin{aligned}
m_{\boldsymbol{\gamma}}^{\mathrm{CR}-\mathrm{PEP}}(y \mid \delta) & =\int \frac{m_{0}^{\mathrm{N}}\left(y^{*} \mid \delta=1\right)}{m_{\boldsymbol{\gamma}}^{\mathrm{N}}\left(y^{*} \mid \delta\right)}\left\{\int f_{\boldsymbol{\gamma}}\left(y \mid \boldsymbol{\beta}_{\boldsymbol{\gamma}}\right) f_{\boldsymbol{\gamma}}\left(y^{*} \mid \boldsymbol{\beta}_{\boldsymbol{\gamma}}, \delta\right) \pi_{\boldsymbol{\gamma}}^{\mathrm{N}}\left(\boldsymbol{\beta}_{\boldsymbol{\gamma}}\right) \mathrm{d} \boldsymbol{\beta}_{\boldsymbol{\gamma}}\right\} \mathrm{d} y^{*} \\
& =\int \mathcal{A}_{\boldsymbol{\gamma}}^{-1} e^{\frac{\delta-1}{\delta} c\left(y^{*}, \phi\right)} \frac{\mathcal{D}\left(y^{*}, 1,1\right)}{\mathcal{D}\left(y^{*}, 1, \delta\right)}\left\{\int f_{\boldsymbol{\gamma}}\left(y \mid \boldsymbol{\beta}_{\boldsymbol{\gamma}}\right) f_{\boldsymbol{\gamma}}\left(y^{*} \mid \boldsymbol{\beta}_{\boldsymbol{\gamma}}, \delta\right) \pi_{\boldsymbol{\gamma}}^{\mathrm{N}}\left(\boldsymbol{\beta}_{\boldsymbol{\gamma}}\right) \mathrm{d} \boldsymbol{\beta}_{\boldsymbol{\gamma}}\right\} \mathrm{d} y^{*} \\
& =e^{c(y, \phi)} \int e^{c\left(y^{*}, \phi\right)} \frac{\mathcal{D}\left(y^{*}, 1,1\right)}{\mathcal{D}\left(y^{*}, 1, \delta\right)} \mathcal{D}\left(\delta y+y^{*}, \delta+1, \delta\right) \mathrm{d} y^{*}
\end{aligned}
$$

which (again) does not depend on the original model formulation $M_{\gamma}$. Hence, for known $\phi$, it is obvious that after integrating out $\delta$ over the hyper-prior $\pi(\delta), m_{\gamma}^{\mathrm{CR}-\mathrm{PEP}}(y)=m_{0}^{\mathrm{CR}-\mathrm{PEP}}(y)$ and this concludes the proof.

If $\phi$ is stochastic and common across models, then the two marginal likelihoods, obtained by integrating out $\phi$ over the common prior $\pi_{0}^{N}(\phi)=\pi_{\gamma}^{N}(\phi)$ for all models $M_{\gamma}$, still coincide.

\section{A.4 Proof of Proposition 4.}

Let us assume known and common across models $\phi_{\gamma}=\phi$ and samples of size $n=p_{\gamma}+1=$ $\sum_{j=1}^{p}+1$. Then the linear predictor is given by $\boldsymbol{\eta}_{\boldsymbol{\gamma}}=\mathrm{X}_{\boldsymbol{\gamma}} \boldsymbol{\beta}_{\boldsymbol{\gamma}}$ where $\mathrm{X}_{\boldsymbol{\gamma}}$ is a matrix of dimension $\left(p_{\gamma}+1\right) \times\left(p_{\gamma}+1\right)$. By considering $n^{*}=n=1+p_{\gamma}$ and further assuming that is invertible, then the prior predictive density for model $M_{0}$ under the baseline prior $\pi_{0}^{N}\left(\beta_{0}\right)$ is given by

$$
m_{0}^{\mathrm{N}}\left(\mathbf{y}^{*} \mid \delta\right)=\int \exp \left(\frac{n^{*} \bar{y}^{*} \vartheta\left(\beta_{0}\right)-n^{*} b\left(\vartheta\left(\beta_{0}\right)\right)}{\delta a(\phi)}+\sum_{i=1}^{n^{*}} \frac{c\left(y_{i}^{*}, \phi\right)}{\delta}\right) \psi\left(\beta_{0}\right) d \beta_{0}
$$


iv

By setting $\eta=\beta_{0}$, we obtain

$$
m_{0}^{\mathrm{N}}\left(\mathbf{y}^{*} \mid \delta\right)=\exp \left(\sum_{i=1}^{n^{*}} \frac{c\left(y_{i}^{*}, \phi\right)}{\delta}\right) \mathcal{D}\left(n^{*} \bar{y}^{*}, n^{*}, \delta\right)
$$

For model $M_{\boldsymbol{\gamma}}$, the prior predictive density under the baseline prior $\pi_{\gamma}^{N}\left(\boldsymbol{\beta}_{\gamma}\right)$ is given by

$$
m_{\boldsymbol{\gamma}}^{\mathrm{N}}\left(\mathbf{y}^{*} \mid \delta\right)=\int \exp \left(\frac{\sum_{i=1}^{n^{*}} y_{i}^{*} \vartheta\left(\eta_{\boldsymbol{\gamma}(i)}\right)-\sum_{i=1}^{n^{*}} b\left(\vartheta\left(\eta_{\boldsymbol{\gamma}(i)}\right)\right)}{\delta a(\phi)}+\sum_{i=1}^{n^{*}} \frac{c\left(y_{i}^{*}, \phi\right)}{\delta}\right) \psi\left(\boldsymbol{\eta}_{\gamma}\right) d \boldsymbol{\beta}_{\boldsymbol{\gamma}}
$$

By setting $\boldsymbol{\eta}=\mathrm{X}_{\boldsymbol{\gamma}} \boldsymbol{\beta}_{\boldsymbol{\gamma}}=\boldsymbol{\eta}_{\gamma}$, we obtain

$$
\begin{aligned}
m_{\boldsymbol{\gamma}}^{\mathrm{N}}\left(\mathbf{y}^{*} \mid \delta\right)= & \exp \left(\sum_{i=1}^{n^{*}} \frac{c\left(y_{i}^{*}, \phi\right)}{\delta}\right) \int \exp \left(\frac{\sum_{i=1}^{n^{*}} y_{i}^{*} \vartheta\left(\eta_{i}\right)-\sum_{i=1}^{n^{*}} b\left(\vartheta\left(\eta_{i}\right)\right)}{\delta a(\phi)}\right) \psi(\boldsymbol{\eta})\left|\mathrm{X}_{\boldsymbol{\gamma}}^{-1}\right| d \boldsymbol{\eta} \\
= & \exp \left(\sum_{i=1}^{n^{*}} \frac{c\left(y_{i}^{*}, \phi\right)}{\delta}\right)\left|\mathrm{X}_{\boldsymbol{\gamma}}^{-1}\right| \mathcal{E}\left(\mathbf{y}^{*}, 1, \delta\right) \\
& \text { where } \mathcal{E}\left(\mathbf{y}^{*}, \omega, \delta\right)=\int \exp \left(\frac{\sum_{i=1}^{n^{*}} y_{i}^{*} \vartheta\left(\eta_{i}\right)-\omega \sum_{i=1}^{n^{*}} b\left(\vartheta\left(\eta_{i}\right)\right)}{\delta a(\phi)}\right) \psi(\boldsymbol{\eta}) d \boldsymbol{\eta} ;
\end{aligned}
$$

note that $\mathcal{E}\left(y^{*}, \omega, \delta\right)=\mathcal{D}\left(y^{*}, \omega, \delta\right)$ for $n^{*}=1$.

The marginal likelihood of $M_{0}$ under the DR-PEP prior, conditional on $\delta$, is given by $m_{0}^{\mathrm{DR}-\mathrm{PEP}}(\mathbf{y} \mid \delta)=m_{0}^{N}(\mathbf{y} \mid \delta)$ while for model $M_{\boldsymbol{\gamma}}$ is given by

$$
\begin{aligned}
m_{\boldsymbol{\gamma}}^{\mathrm{DR}-\mathrm{PEP}}(\mathbf{y} \mid \delta) & =\mathcal{C}_{0}^{-1} \int\left|\mathrm{X}_{\boldsymbol{\gamma}}\right| \frac{\mathcal{D}\left(n^{*} \bar{y}^{*}, n^{*}, \delta\right)}{\mathcal{E}\left(\mathbf{y}^{*}, 1, \delta\right)}\left\{\int f_{\boldsymbol{\gamma}}\left(y \mid \boldsymbol{\beta}_{\boldsymbol{\gamma}}\right) f_{\boldsymbol{\gamma}}\left(y^{*} \mid \boldsymbol{\beta}_{\boldsymbol{\gamma}}, \delta\right) \pi_{\boldsymbol{\gamma}}^{\mathrm{N}}\left(\boldsymbol{\beta}_{\boldsymbol{\gamma}}\right) \mathrm{d} \boldsymbol{\beta}_{\boldsymbol{\gamma}}\right\} \mathrm{d} \mathbf{y}^{*} \\
& =\mathcal{C}_{0}^{-1}\left|\mathrm{X}_{\boldsymbol{\gamma}}\right| \int \frac{\mathcal{D}\left(n^{*} \bar{y}^{*}, n^{*}, \delta\right)}{\mathcal{E}\left(\mathbf{y}^{*}, 1, \delta\right)}\left\{\int f_{\boldsymbol{\gamma}}\left(y \mid \boldsymbol{\beta}_{\boldsymbol{\gamma}}\right) f_{\boldsymbol{\gamma}}\left(y^{*} \mid \boldsymbol{\beta}_{\boldsymbol{\gamma}}, \delta\right) \pi_{\boldsymbol{\gamma}}^{\mathrm{N}}\left(\boldsymbol{\beta}_{\boldsymbol{\gamma}}\right) \mathrm{d} \boldsymbol{\beta}_{\boldsymbol{\gamma}}\right\} \mathrm{d} \mathbf{y}^{*} \\
& =\mathcal{C}_{0}^{-1}\left|\mathrm{X}_{\boldsymbol{\gamma}}\right| \int \frac{\mathcal{D}\left(n^{*} \bar{y}^{*}, n^{*}, \delta\right)}{\mathcal{E}\left(\mathbf{y}^{*}, 1, \delta\right)} \exp \left(\sum_{i=1}^{n^{*}} c\left(y_{i}, \phi\right)+\sum_{i=1}^{n^{*}} \frac{c\left(y_{i}^{*}, \phi\right)}{\delta}\right) \\
& \times\left|\mathrm{X}_{\boldsymbol{\gamma}}\right|^{-1} \mathcal{E}\left(\delta \mathbf{y}+\mathbf{y}^{*}, \delta+1, \delta\right) \mathrm{d} \mathbf{y}^{*} \\
& =\mathcal{C}_{0}^{-1} \int \prod_{i=1}^{n^{*}} e^{c\left(y_{i}, \phi\right)+\frac{c\left(y_{i}^{*}, \phi\right)}{\delta}} \mathcal{D}\left(n^{*} \bar{y}^{*}, n^{*}, \delta\right) \frac{\mathcal{E}\left(\delta \mathbf{y}+\mathbf{y}^{*}, \delta+1, \delta\right)}{\mathcal{E}\left(\mathbf{y}^{*}, 1, \delta\right)} \mathrm{d} \mathbf{y}^{*}
\end{aligned}
$$

which coincides for any model of the same dimension with training samples of the same size. Hence, for known $\phi$, this concludes the proof for the (fixed $\delta$ ) DR-PEP prior. It is obvious that for random $\delta$ integrating out the above marginal likelihoods over any hyper-prior $\pi(\delta)$ will result to $m_{\boldsymbol{\gamma}}^{\mathrm{DR}-\mathrm{PEP}}(\mathbf{y})=m_{0}^{\mathrm{DR}-\mathrm{PEP}}(\mathbf{y})$ and this concludes the proof for hyper- $\delta$ DR-PEP.

Finally if $\phi$ is stochastic and common across models, then the two marginal likelihoods (under fixed or random $\delta$ ), obtained by integrating out $\phi$ over the common prior $\pi_{0}^{N}(\phi)=$ $\pi_{\gamma}^{N}(\phi)$ for all models $M_{\gamma}$, still coincide. 


\section{Appendix B: The PEP-GVS algorithm}

\section{B.1 Implementation details}

Concerning the binary inclusion indicators $\gamma_{j}$, the conditional posterior distribution $\pi\left(\gamma_{j} \mid \boldsymbol{\beta}, \gamma_{\backslash j}, \mathbf{y}^{*}, \mathbf{y}, \delta\right)$ is a Bernoulli distribution with success probability $O_{j} /\left(1+O_{j}\right)$ and

$$
O_{j}=\frac{f_{\gamma_{j_{1}}}\left(\mathbf{y} \mid \boldsymbol{\beta}_{\gamma_{j_{1}}}\right)}{f_{\gamma_{j_{0}}}\left(\mathbf{y} \mid \boldsymbol{\beta}_{\gamma_{j_{0}}}\right)}\left[\frac{f_{\gamma_{j_{1}}}\left(\mathbf{y}^{*} \mid \boldsymbol{\beta}_{\gamma_{j_{1}}}\right)}{f_{\gamma_{j_{0}}}\left(\mathbf{y}^{*} \mid \boldsymbol{\beta}_{\gamma_{j_{0}}}\right)}\right]^{1 / \delta} \frac{\pi_{\boldsymbol{\gamma}_{j_{1}}}^{\mathrm{N}}(\boldsymbol{\beta})}{\pi_{\boldsymbol{\gamma}_{j_{0}}}^{\mathrm{N}}(\boldsymbol{\beta})} \frac{m_{\boldsymbol{\gamma}_{j_{0}}}^{\mathrm{N}}\left(\mathbf{y}^{*} \mid \delta\right)}{m_{\boldsymbol{\gamma}_{j_{1}}}^{\mathrm{N}}\left(\mathbf{y}^{*} \mid \delta\right)} \frac{\pi\left(\boldsymbol{\gamma}_{j_{1}}\right)}{\pi\left(\boldsymbol{\gamma}_{j_{0}}\right)},
$$

where $\gamma_{j_{1}}=\left(\gamma_{j}=1, \gamma_{\backslash j}\right), \gamma_{j_{0}}=\left(\gamma_{j}=0, \gamma_{\backslash j}\right)$ and $\pi_{\gamma}^{\mathrm{N}}(\boldsymbol{\beta})=\pi_{\gamma}^{\mathrm{N}}\left(\boldsymbol{\beta}_{\boldsymbol{\gamma}}\right) \pi_{\gamma}^{\mathrm{N}}\left(\boldsymbol{\beta}_{\backslash \boldsymbol{\gamma}}\right)$ for $\boldsymbol{\gamma} \in$ $\left\{\gamma_{j_{1}}, \gamma_{j_{0}}\right\}$. All of the quantities involved in (B.1) are available in closed form expressions except of the marginal likelihood $m_{\gamma}^{\mathrm{N}}\left(\mathbf{y}^{*} \mid \delta\right)$. The latter is estimated through the following Laplace approximation

$$
\widehat{m}_{\gamma}^{\mathrm{N}}\left(\mathbf{y}^{*} \mid \delta\right)=(2 \pi \delta)^{d_{\gamma} / 2}\left|\mathbf{X}_{\gamma}^{T} \mathbf{W}_{\boldsymbol{\gamma}}\left(\widehat{\boldsymbol{\beta}}_{\gamma}^{*}\right) \mathbf{X}_{\boldsymbol{\gamma}}\right|^{-1 / 2} f_{\boldsymbol{\gamma}}\left(\mathbf{y}^{*} \mid \widehat{\boldsymbol{\beta}}_{\gamma}^{*}\right)^{1 / \delta} \pi_{\gamma}^{\mathrm{N}}\left(\widehat{\boldsymbol{\beta}}_{\gamma}^{*}\right),
$$

where $\widehat{\boldsymbol{\beta}}_{\gamma}^{*}$ is the MLE for data $\mathbf{y}^{*}$ given the configuration of $\boldsymbol{\gamma}$ and $\delta\left[\mathbf{X}_{\gamma}^{T} \mathbf{W}_{\boldsymbol{\gamma}}\left(\widehat{\boldsymbol{\beta}}_{\gamma}^{*}\right) \mathbf{X}_{\gamma}\right]^{-1}$ is equal to minus the inverse Hessian matrix evaluated at $\widehat{\boldsymbol{\beta}}_{\gamma}^{*}$. Under a Jeffreys baseline prior for $\boldsymbol{\beta}_{\boldsymbol{\gamma}}$, the Laplace approximation simplifies to $\widehat{m}_{\boldsymbol{\gamma}}^{\mathrm{N}}\left(\mathbf{y}^{*} \mid \delta\right)=(2 \pi \delta)^{d_{\boldsymbol{\gamma}} / 2} f_{\boldsymbol{\gamma}}\left(\mathbf{y}^{*} \mid \widehat{\boldsymbol{\beta}}_{\gamma}^{*}\right)^{1 / \delta}$. A comparison with respect to numerical integration, in terms of the the marginal likelihood log-ratios, is provided in Appendix C.

For the active effects $\boldsymbol{\beta}_{\gamma}$ of model $M_{\gamma}$ and the intercept term $\beta_{0}$ of the reference model $M_{0}$, we use independence sampler M-H steps. Specifically, for $\boldsymbol{\beta}_{\boldsymbol{\gamma}}$ we generate new candidate values as

$$
\boldsymbol{\beta}_{\gamma}^{\prime} \sim q\left(\boldsymbol{\beta}_{\gamma}^{\prime}\right) \equiv \mathrm{N}_{d_{\gamma}}\left(\widehat{\boldsymbol{\beta}}_{\gamma}^{\text {all }}, \widehat{\Sigma}_{\boldsymbol{\beta}_{\gamma}^{\text {all }}}\right),
$$

where $\widehat{\boldsymbol{\beta}}_{\gamma}^{\text {all }}$ is the ML estimate from a weighted regression on $\mathbf{y}^{\text {all }}=\left(\mathbf{y}, \mathbf{y}^{*}\right)^{T}$, using weights $\mathbf{w}^{\text {all }}=\left(\mathbf{1}_{n}, \mathbf{1}_{n} \delta^{-1}\right)^{T}$, and $\widehat{\Sigma}_{\boldsymbol{\beta}_{\gamma}^{\text {all }}}$ is the estimated variance-covariance matrix of $\widehat{\boldsymbol{\beta}}_{\gamma}^{\text {all }}$. The proposed move is accepted with probability

$$
A_{\boldsymbol{\beta}_{\gamma}}=\min \left[1, \frac{f_{\boldsymbol{\gamma}}\left(\mathbf{y} \mid \boldsymbol{\beta}_{\gamma}^{\prime}\right)}{f_{\boldsymbol{\gamma}}\left(\mathbf{y} \mid \boldsymbol{\beta}_{\gamma}\right)}\left(\frac{f_{\boldsymbol{\gamma}}\left(\mathbf{y}^{*} \mid \boldsymbol{\beta}_{\gamma}^{\prime}\right)}{f_{\boldsymbol{\gamma}}\left(\mathbf{y}^{*} \mid \boldsymbol{\beta}_{\gamma}\right)}\right)^{1 / \delta} \frac{\pi_{\gamma}^{\mathrm{N}}\left(\boldsymbol{\beta}_{\gamma}^{\prime}\right) q\left(\boldsymbol{\beta}_{\gamma}\right)}{\pi_{\gamma}^{\mathrm{N}}\left(\boldsymbol{\beta}_{\boldsymbol{\gamma}}\right) q\left(\boldsymbol{\beta}_{\gamma}^{\prime}\right)}\right],
$$

where $\boldsymbol{\beta}_{\boldsymbol{\gamma}}$ denotes the current value of the chain. The proposal distribution of $\beta_{0}$ is $q\left(\beta_{0}\right)=$ $\mathrm{N}\left(\widehat{\beta_{0}}, \psi \widehat{\sigma}_{\beta_{0}}^{2}\right)$ with $\widehat{\beta_{0}}$ and $\widehat{\sigma}_{\beta_{0}}$ being the respective ML estimate of $\beta_{0}$ and the standard error of $\widehat{\beta_{0}}$ from the null model with response data $\mathbf{y}^{*}$. The proposed move is accepted with the usual $\mathrm{M}-\mathrm{H}$ transition probability where the likelihood of the reference model is raised to the power of $1 / \psi$. Note that no specific fine tuning is required for the proposal distributions of $\boldsymbol{\beta}_{\boldsymbol{\gamma}}$ and $\beta_{0}$.

For the generation of the imaginary data we propose candidate values $\mathbf{y}^{*^{\prime}}$ from a proposal distribution $q\left(\mathbf{y}^{*^{\prime}}\right)$ and accept the proposed move with probability

$$
A_{\mathbf{y}^{*}}=\min \left[1,\left(\frac{f_{\boldsymbol{\gamma}}\left(\mathbf{y}^{*^{\prime}} \mid \boldsymbol{\beta}_{\boldsymbol{\gamma}}\right)}{f_{\boldsymbol{\gamma}}\left(\mathbf{y}^{*} \mid \boldsymbol{\beta}_{\boldsymbol{\gamma}}\right)}\right)^{1 / \delta}\left(\frac{f_{0}\left(\mathbf{y}^{*^{\prime}} \mid \beta_{0}\right)}{f_{0}\left(\mathbf{y}^{*} \mid \beta_{0}\right)}\right)^{1 / \psi} \frac{\widehat{m}_{\boldsymbol{\gamma}}^{\mathrm{N}}\left(\mathbf{y}^{*} \mid \delta\right)}{\widehat{m}_{\boldsymbol{\gamma}}^{\mathrm{N}}\left(\mathbf{y}^{*^{\prime}} \mid \delta\right)} \frac{q\left(\mathbf{y}^{*}\right)}{q\left(\mathbf{y}^{*^{\prime}}\right)}\right],
$$


where the marginal likelihood estimates are obtained through (B.2) and $\mathbf{y}^{*}$ denotes the current value of the chain. The joint proposal density is formed by the product of independent distributions, i.e. $q\left(\mathbf{y}^{*}\right)=\prod_{i=1}^{n^{*}} q\left(y_{i}^{*}\right)$, where the proposal of each imaginary observation $y_{i}^{*}$ is constructed by combining the two likelihood components of the PEP prior. Hence, for the logistic regression model we use

$$
q\left(y_{i}^{*}\right) \equiv \operatorname{Binomial}\left(N_{i}, \pi_{i}^{*}\right) \text { with } \pi_{i}^{*}=\frac{\pi_{0}^{1 / \psi} \pi_{\gamma(i)}^{1 / \delta}}{\pi_{0}^{1 / \psi} \pi_{\gamma(i)}^{1 / \delta}+\left(1-\pi_{0}\right)^{1 / \psi}\left(1-\pi_{\gamma(i)}\right)^{1 / \delta}},
$$

where $\pi_{0}=\left(1+\exp \left(-\beta_{0}\right)\right)^{-1}, \pi_{\gamma(i)}=\left(1+\exp \left(-\mathbf{X}_{\boldsymbol{\gamma}(i)} \boldsymbol{\beta}_{\gamma}\right)\right)^{-1}$ and $N_{i}$ denotes the number of trials of the observed data. Equivalently, for Poisson regression models we consider

$$
q\left(y_{i}^{*}\right) \equiv \text { Poisson }\left(\lambda_{0} \lambda_{\gamma(i)}^{1 / \delta}\right)
$$

for the CR-PEP prior; where $\lambda_{0}=\exp \left(\beta_{0}\right)$ and $\lambda_{\boldsymbol{\gamma}(i)}=\exp \left(\mathbf{X}_{\boldsymbol{\gamma}(i)} \boldsymbol{\beta}_{\boldsymbol{\gamma}}\right)$. For the DR-PEP prior, the corresponding choice of a Poisson proposal with mean $\left(\lambda_{0} \lambda_{\gamma}\right)^{1 / \delta}$ was not found to be efficient in practice. Therefore, we use instead a Poisson random-walk proposal with mean equal to the value of $y_{i}^{*}$ at the current iteration.

Finally, when $\delta$ is random the corresponding full conditionals we need to sample from are

$$
\begin{aligned}
& \pi^{\mathrm{CR}-\mathrm{PEP}}\left(\delta \mid \boldsymbol{\beta}_{\boldsymbol{\gamma}}, \beta_{0}, \boldsymbol{\gamma}, \mathbf{y}^{*}, \mathbf{y}\right) \propto \frac{f_{\boldsymbol{\gamma}}\left(\mathbf{y}^{*} \mid \boldsymbol{\beta}_{\boldsymbol{\gamma}}\right)^{1 / \delta} \pi(\delta)}{m_{\boldsymbol{\gamma}}^{\mathrm{N}}\left(\mathbf{y}^{*} \mid \delta\right)}, \\
& \pi^{\mathrm{DR}-\mathrm{PEP}}\left(\delta \mid \boldsymbol{\beta}_{\boldsymbol{\gamma}}, \beta_{0}, \boldsymbol{\gamma}, \mathbf{y}^{*}, \mathbf{y}\right) \propto \frac{f_{\boldsymbol{\gamma}}\left(\mathbf{y}^{*} \mid \boldsymbol{\beta}_{\boldsymbol{\gamma}}\right)^{1 / \delta} f_{0}\left(\mathbf{y}^{*} \mid \beta_{0}\right)^{1 / \delta} \pi(\delta)}{m_{\boldsymbol{\gamma}}^{\mathrm{N}}\left(\mathbf{y}^{*} \mid \delta\right)} .
\end{aligned}
$$

Looking at the above expressions, a subtle point is that $\delta$ is not directly linked to the actual data $\mathbf{y}$; however, it is linked indirectly via the posterior values of the parameters of models $M_{\gamma}$ (for both approaches) and $M_{0}$ (for the DR-PEP prior). Sampling from (B.3) or (B.4) is achieved by adding one simple step (after Step 7) in the PEP-GVS algorithm described in Section 3.2. Specifically, we use a random walk M-H step where we propose a candidate value $\delta^{\prime}$ from $q\left(\delta^{\prime} \mid \delta\right)=\operatorname{Gamma}\left(\delta^{2} / s_{\delta}^{2}, \delta / s_{\delta}^{2}\right)$, which has mean equal to the current value $\delta$ and variance $s_{\delta}^{2}$. The latter is a tuning parameter which can be specified appropriately in order to have an acceptance rate between 0.2 and 0.5 . The value of $s_{\delta}^{2}=\delta$ proved to be efficient in the examples presented in Section 7. Given this proposal, the new candidate $\delta^{\prime}$ is accepted with probability $A_{\delta}^{\prime}=\min \left(1, A_{\delta}\right)$, with $A_{\delta}$ given by

$$
A_{\delta}=\left(\frac{\delta}{\delta^{\prime}}\right)^{d_{\boldsymbol{\gamma}} / 2}\left[\frac{f_{\boldsymbol{\gamma}}\left(\mathbf{y}^{*} \mid \boldsymbol{\beta}_{\boldsymbol{\gamma}}\right)}{f_{\boldsymbol{\gamma}}\left(\mathbf{y}^{*} \mid \widehat{\boldsymbol{\beta}}_{\boldsymbol{\gamma}}^{*}\right)}\right]^{\left\{\frac{1}{\delta^{\prime}}-\frac{1}{\delta}\right\}} f_{0}\left(\mathbf{y}^{*} \mid \beta_{0}\right)\left\{\frac{1}{\psi^{\prime}}-\frac{1}{\psi}\right\} \frac{\pi\left(\delta^{\prime}\right)}{\pi(\delta)} \frac{q\left(\delta \mid \delta^{\prime}\right)}{q\left(\delta^{\prime} \mid \delta\right)},
$$

where $\psi^{\prime}=\psi=1$ for the CR-PEP prior and $\psi^{\prime}=\delta^{\prime}, \psi=\delta$ for the DR-PEP prior; $\widehat{\boldsymbol{\beta}}_{\gamma}^{*}$ is the MLE for $\boldsymbol{\beta}_{\gamma}$ using data $\mathbf{y}^{*}$. 


\section{B.2 An analytic description}

Given the posterior distribution in Eq. 26, with $\psi=1$ for the CR-PEP prior and $\psi=\delta$ for the DR-PEP prior, the PEP-GVS sampler proceeds as follows:

A. Set starting values $\boldsymbol{\gamma}^{(0)}, \boldsymbol{\beta}^{(0)}=\left(\boldsymbol{\beta}_{\boldsymbol{\gamma}}^{(0)}, \boldsymbol{\beta}_{\backslash \boldsymbol{\gamma}}^{(0)}\right), \beta_{0}^{(0)}$ and $\mathbf{y}^{*(0)}$. For fixed $\delta$ set $\delta=n$, for random $\delta$ set starting starting value $\delta^{(0)}$.

B. For iterations $t=1,2, \ldots, N$ :

Step 1: Sampling of $\gamma_{j}^{(t)}$, for $j=1,2, \ldots, p$, given the current state of $\boldsymbol{\beta}_{\boldsymbol{\gamma}}, \boldsymbol{\beta}_{\backslash \boldsymbol{\gamma}}, \boldsymbol{\gamma}_{\backslash j}, \mathbf{y}^{*}$ and $\delta$.

(a) Calculate the MLEs under $\gamma_{j_{1}}=\left(\gamma_{j}=1, \gamma_{\backslash j}\right), \gamma_{j_{0}}=\left(\gamma_{j}=0, \gamma_{\backslash j}\right)$ and compute the Laplace approximations $\widehat{m}_{\gamma_{j_{1}}}^{\mathrm{N}}\left(\mathbf{y}^{*} \mid \delta\right), \widehat{m}_{\gamma_{j_{0}}}^{\mathrm{N}}\left(\mathbf{y}^{*} \mid \delta\right)$ through Eq. B.2.

(b) Evaluate the odds:

$$
\begin{aligned}
O_{j}= & \frac{f_{\boldsymbol{\gamma}_{j_{1}}}\left(\mathbf{y} \mid \boldsymbol{\beta}_{\boldsymbol{\gamma}_{j_{1}}}\right)}{f_{\boldsymbol{\gamma}_{j_{0}}}\left(\mathbf{y} \mid \boldsymbol{\beta}_{\boldsymbol{\gamma}_{0}}\right)}\left[\frac{f_{\boldsymbol{\gamma}_{j_{1}}}\left(\mathbf{y}^{*} \mid \boldsymbol{\beta}_{\boldsymbol{\gamma}_{j_{1}}}\right)}{f_{\boldsymbol{\gamma}_{j_{0}}}\left(\mathbf{y}^{*} \mid \boldsymbol{\beta}_{\boldsymbol{\gamma}_{j_{0}}}\right)}\right]^{1 / \delta} \frac{\pi_{\boldsymbol{\gamma}_{j_{1}}}^{\mathrm{N}}\left(\boldsymbol{\beta}_{\boldsymbol{\gamma}_{j_{1}}}\right)}{\pi_{\boldsymbol{\gamma}_{j_{0}}}^{\mathrm{N}}\left(\boldsymbol{\beta}_{\boldsymbol{\gamma}_{j_{0}}}\right)} \frac{\pi_{\boldsymbol{\gamma}_{j_{1}}}^{\mathrm{N}}\left(\boldsymbol{\beta}_{\backslash \boldsymbol{\gamma}_{j_{1}}}\right)}{\pi_{\boldsymbol{\gamma}_{j_{0}}}^{\mathrm{N}}\left(\boldsymbol{\beta}_{\backslash \boldsymbol{\gamma}_{j_{0}}}\right)} \\
& \times \frac{\widehat{m}_{\boldsymbol{\gamma}_{j_{0}}}^{\mathrm{N}}\left(\mathbf{y}^{*} \mid \delta\right)}{\widehat{m}_{\boldsymbol{\gamma}_{j_{1}}}^{\mathrm{N}}\left(\mathbf{y}^{*} \mid \delta\right)} \frac{\pi\left(\boldsymbol{\gamma}_{j_{1}}\right)}{\pi\left(\boldsymbol{\gamma}_{j_{0}}\right)}
\end{aligned}
$$

(c) Sample $\gamma_{j}^{\prime} \sim \operatorname{Bernoulli}\left(\frac{O_{j}}{1+O_{j}}\right)$ and set $\gamma_{j}^{(t)}=\gamma_{j}^{\prime}$ with probability equal to 1 .

Step 2: Update $\boldsymbol{\beta}^{(t-1)}=\left(\boldsymbol{\beta}_{\boldsymbol{\gamma}}^{(t-1)}, \boldsymbol{\beta}_{\backslash \boldsymbol{\gamma}}^{(t-1)}\right)$ based on the current configuration of $\boldsymbol{\gamma}$.

Step 3: Sampling of $\boldsymbol{\beta}_{\boldsymbol{\gamma}}^{(t)}$ given the current state of $\gamma, \mathbf{y}^{*}$ and $\delta$.

(a) Generate $\boldsymbol{\beta}_{\boldsymbol{\gamma}}^{\prime}$ from the proposal distribution $q\left(\boldsymbol{\beta}_{\boldsymbol{\gamma}}^{\prime}\right)=\mathrm{N}_{d_{\boldsymbol{\gamma}}}\left(\widehat{\boldsymbol{\beta}}_{\boldsymbol{\gamma}}^{\text {all }}, \widehat{\Sigma}_{\boldsymbol{\beta}_{\boldsymbol{\gamma}}^{\text {all }}}\right)$, where $\widehat{\boldsymbol{\beta}}_{\boldsymbol{\gamma}}^{\text {all }}$ is the ML estimate from a weighted regression on $\mathbf{y}^{\text {all }}=\left(\mathbf{y}, \mathbf{y}^{*}\right)^{T}$, using weights $\mathbf{w}^{\text {all }}=\left(\mathbf{1}_{n}, \mathbf{1}_{n} \delta^{-1}\right)^{T}$, and $\widehat{\Sigma}_{\boldsymbol{\beta}_{\boldsymbol{\gamma}}^{\text {all }}}$ is the estimated variance-covariance matrix of $\widehat{\boldsymbol{\beta}}_{\gamma}^{\text {all }}$

(b) Calculate the probability of accepting the proposed move:

$$
A_{\boldsymbol{\beta}_{\boldsymbol{\gamma}}}=1 \wedge\left[\frac{f_{\boldsymbol{\gamma}}\left(\mathbf{y} \mid \boldsymbol{\beta}_{\boldsymbol{\gamma}}^{\prime}\right)}{f_{\boldsymbol{\gamma}}\left(\mathbf{y} \mid \boldsymbol{\beta}_{\boldsymbol{\gamma}}^{(t-1)}\right)}\left\{\frac{f_{\boldsymbol{\gamma}}\left(\mathbf{y}^{*} \mid \boldsymbol{\beta}_{\boldsymbol{\gamma}}^{\prime}\right)}{f_{\boldsymbol{\gamma}}\left(\mathbf{y}^{*} \mid \boldsymbol{\beta}_{\boldsymbol{\gamma}}^{(t-1)}\right)}\right\}^{1 / \delta} \frac{\pi_{\boldsymbol{\gamma}}^{\mathrm{N}}\left(\boldsymbol{\beta}_{\boldsymbol{\gamma}}^{\prime}\right)}{\pi_{\boldsymbol{\gamma}}^{\mathrm{N}}\left(\boldsymbol{\beta}_{\boldsymbol{\gamma}}^{(t-1)}\right)} \frac{q\left(\boldsymbol{\beta}_{\boldsymbol{\gamma}}^{(t-1)}\right)}{q\left(\boldsymbol{\beta}_{\boldsymbol{\gamma}}^{\prime}\right)}\right] .
$$

(c) Set $\boldsymbol{\beta}_{\boldsymbol{\gamma}}^{(t)}=\left\{\begin{array}{l}\boldsymbol{\beta}_{\boldsymbol{\gamma}}^{\prime} \text { with probability } A_{\boldsymbol{\beta}_{\boldsymbol{\gamma}}}, \\ \boldsymbol{\beta}_{\boldsymbol{\gamma}}^{(t-1)} \text { with probability } 1-A_{\boldsymbol{\beta}_{\boldsymbol{\gamma}}} \text {. }\end{array}\right.$

Step 4: Sampling of $\boldsymbol{\beta}_{\backslash \gamma}^{(t)}$ given the current state of $\gamma$.

(a) Generate $\boldsymbol{\beta}_{\backslash \gamma}^{\prime}$ from the pseudo-prior $\pi_{\boldsymbol{\gamma}}^{\mathrm{N}}\left(\boldsymbol{\beta}_{\backslash \gamma}^{\prime}\right)=\mathrm{N}_{d_{\backslash \gamma}}\left(\widehat{\boldsymbol{\beta}}_{\backslash \boldsymbol{\gamma}}, \mathbf{I}_{d_{\backslash \gamma}} \widehat{\sigma}_{\boldsymbol{\beta}_{\backslash \gamma}}^{2}\right)$, where $\widehat{\boldsymbol{\beta}}_{\backslash \boldsymbol{\gamma}}$ and $\widehat{\sigma}_{\boldsymbol{\beta}_{\backslash \gamma}}$ are the respective MLEs and corresponding standard errors of $\boldsymbol{\beta}_{\backslash \gamma}$ from the full model given data $\mathbf{y}$. 
viii

(b) Set $\boldsymbol{\beta}_{\backslash \gamma}^{(t)}=\boldsymbol{\beta}_{\backslash \gamma}^{\prime}$ with probability equal to 1 .

Step 5: Sampling of $\beta_{0}^{(t)}$ given the current state of $\mathbf{y}^{*}$ and $\delta$.

(a) Generate $\beta_{0}^{\prime}$ from the proposal distribution $q\left(\beta_{0}^{\prime}\right)=\mathrm{N}\left(\widehat{\beta}_{0}, \psi \widehat{\sigma}_{\beta_{0}}^{2}\right)$, where $\widehat{\beta}_{0}$ and $\widehat{\sigma}_{\beta_{0}}$ are the respective MLE of $\beta_{0}$ and the standard error of $\widehat{\beta}_{0}$ from the null model given data $\mathbf{y}^{*}$.

(b) Calculate the probability of accepting the proposed move:

$$
A_{\beta_{0}}=1 \wedge\left[\left\{\frac{f_{0}\left(\mathbf{y}^{*} \mid \beta_{0}^{\prime}\right)}{f_{0}\left(\mathbf{y}^{*} \mid \beta_{0}^{(t-1)}\right)}\right\}^{1 / \psi} \frac{\pi_{0}^{\mathrm{N}}\left(\beta_{0}^{\prime}\right)}{\pi_{0}^{\mathrm{N}}\left(\beta_{0}^{(t-1)}\right)} \frac{q\left(\beta_{0}^{(t-1)}\right)}{q\left(\beta_{0}^{\prime}\right)}\right] .
$$

(c) Set $\beta_{0}^{(t)}= \begin{cases}\beta_{0}^{\prime} & \text { with probability } A_{\beta_{0}}, \\ \beta_{0}^{(t-1)} & \text { with probability } 1-A_{\beta_{0}} .\end{cases}$

Step 6: Sampling of $\mathbf{y}^{*(t)}$ given the current state of $\boldsymbol{\beta}_{\boldsymbol{\gamma}}, \beta_{0}, \boldsymbol{\gamma}$ and $\delta$.

(a) Generate $\mathbf{y}^{*^{\prime}}$ from a proposal distribution $q\left(\mathbf{y}^{*^{\prime}}\right)$; for details about this proposal, see the remarks in the subsection which immediately follows the description of the algorithm.

(b) Calculate the MLEs given $\mathbf{y}^{*(t-1)}$ and $\mathbf{y}^{*^{\prime}}$ and compute the Laplace approximations $\widehat{m}_{\boldsymbol{\gamma}}^{\mathrm{N}}\left(\mathbf{y}^{*(t-1)} \mid \delta\right)$ and $\widehat{m}_{\boldsymbol{\gamma}}^{\mathrm{N}}\left(\mathbf{y}^{*^{\prime}} \mid \delta\right)$ through Eq. B.2.

(c) Calculate the probability $A_{\mathbf{y}^{*}}$ of accepting the proposed move equal to

$$
1 \wedge\left[\left\{\frac{f_{\gamma}\left(\mathbf{y}^{*^{\prime}} \mid \boldsymbol{\beta}_{\gamma}\right)}{f_{\gamma}\left(\mathbf{y}^{*(t-1)} \mid \boldsymbol{\beta}_{\gamma}\right)}\right\}^{1 / \delta}\left\{\frac{f_{0}\left(\mathbf{y}^{*^{\prime}} \mid \beta_{0}\right)}{f_{0}\left(\mathbf{y}^{*(t-1)} \mid \beta_{0}\right)}\right\}^{1 / \psi} \frac{\widehat{m}_{\gamma}^{\mathrm{N}}\left(\mathbf{y}^{*(t-1)} \mid \delta\right)}{\widehat{m}_{\gamma}^{\mathrm{N}}\left(\mathbf{y}^{*^{\prime}} \mid \delta\right)} \frac{q\left(\mathbf{y}^{*(t-1)}\right)}{q\left(\mathbf{y}^{*^{\prime}}\right)}\right] .
$$

(d) Set $\mathbf{y}^{*(t)}= \begin{cases}\mathbf{y}^{* \prime} & \text { with probability } A_{\mathbf{y}^{*}}, \\ \mathbf{y}^{*(t-1)} & \text { with probability } 1-A_{\mathbf{y}^{*}}\end{cases}$

Step 7: Sampling of $\delta^{(t)}$ given the current state of $\boldsymbol{\beta}_{\boldsymbol{\gamma}}, \beta_{0}$ and $\boldsymbol{\gamma}$.

(a) If $\delta$ is fixed at $n$ go to Step 1, else implement (b)-(e) of Step 7.

(b) Generate $\delta^{\prime}$ from the proposal distribution $q\left(\delta^{\prime} \mid \delta^{(t-1)}\right)=\operatorname{Gamma}\left(a^{\prime}, b^{\prime}\right)$ with $a^{\prime}=\delta^{(t-1)^{2}} / s_{\delta}^{2}$ and $b^{\prime}=\delta^{(t-1)} / s_{\delta}^{2}$.

(d) Calculate the probability of accepting the proposed move:

$$
\begin{aligned}
A_{\delta}=1 \wedge\{ & {\left[\left(\frac{\delta^{(t-1)}}{\delta^{\prime}}\right)^{d_{\gamma} / 2}\left(\frac{f_{\boldsymbol{\gamma}}\left(\mathbf{y}^{*} \mid \boldsymbol{\beta}_{\gamma}\right)}{f_{\boldsymbol{\gamma}}\left(\mathbf{y}^{*} \mid \widehat{\boldsymbol{\beta}}_{\gamma}^{*}\right)}\right)^{\left\{\frac{1}{\delta^{\prime}}-\frac{1}{\delta^{(t-1)}}\right\}}\right] \times } \\
& \left.\times\left[f_{0}\left(\mathbf{y}^{*} \mid \beta_{0}\right)\left\{\frac{1}{\psi^{\prime}}-\frac{1}{\psi^{(t-1)}}\right\} \frac{\pi\left(\delta^{\prime}\right)}{\pi\left(\delta^{(t-1)}\right)} \frac{q\left(\delta^{(t-1)} \mid \delta^{\prime}\right)}{q\left(\delta^{\prime} \mid \delta^{(t-1)}\right)}\right]\right\},
\end{aligned}
$$

where $\psi^{\prime}=\psi^{(t-1)}=1$ for the CR-PEP prior and $\psi^{\prime}=\delta^{\prime}, \psi^{(t-1)}=\delta^{(t-1)}$ for the DR-PEP prior. 


$$
\text { (e) } \operatorname{Set} \delta^{(t)}= \begin{cases}\delta^{\prime} & \text { with probability } A_{\delta} \\ \delta^{(t-1)} & \text { with probability } 1-A_{\delta}\end{cases}
$$

C. Repeat the steps in B until convergence.

Suggested proposals for Step 6: For $\mathbf{y}^{*}$ we recommend the following proposals depending on the likelihood of the model and on the PEP prior that is used:

i) For logistic regression a product binomial proposal distribution given by

$$
q\left(\mathbf{y}^{*}\right)=\prod_{i=1}^{n^{*}} \operatorname{Binomial}\left(N_{i}, \pi_{i}^{*}\right) \text { with } \pi_{i}^{*}=\frac{\pi_{0}^{1 / \psi} \pi_{\gamma(i)}^{1 / \delta}}{\pi_{0}^{1 / \psi} \pi_{\gamma(i)}^{1 / \delta}+\left(1-\pi_{0}\right)^{1 / \psi}\left(1-\pi_{\gamma(i)}\right)^{1 / \delta}}
$$

where $\pi_{0}=\left\{1+\exp \left(-\beta_{0}\right)\right\}^{-1}, \pi_{\boldsymbol{\gamma}(i)}=\left\{1+\exp \left(-\mathbf{X}_{\boldsymbol{\gamma}(i)} \boldsymbol{\beta}_{\boldsymbol{\gamma}}\right)\right\}^{-1}$ and $N_{i}$ denotes the number of trials of the observed data.

ii) For Poisson regression a product Poisson proposal distribution given by $q\left(\mathbf{y}^{*}\right)=\prod_{i=1}^{n^{*}} \operatorname{Pois}\left(\lambda_{i}^{*}\right)$.

For the CR-PEP prior $\lambda_{i}^{*}=\lambda_{0} \lambda_{\boldsymbol{\gamma}(i)}^{1 / \delta}$, where $\lambda_{0}=\exp \left(\beta_{0}\right)$ and $\lambda_{\boldsymbol{\gamma}(i)}=\exp \left(\mathbf{X}_{\boldsymbol{\gamma}(i)} \boldsymbol{\beta}_{\boldsymbol{\gamma}}\right)$.

For the DR-PEP prior we utilize a random-walk proposal, i.e. $\lambda_{i}^{*}=y_{i}^{*(t-1)}$. 


\section{Appendix C: Laplace Approximation vs. Numerical Integration}

The Laplace approximation given in Eq. B.2 is used during the MCMC algorithm in order to calculate the marginal likelihood ratios (MLR) appearing in Step 1(b) and in Step 6(c) of the GVS-PEP algorithm of Appendix B.2. For instance, in Step 6(c) at iteration $t$ we use the Laplace approximation to evaluate the quantity

$$
\operatorname{MLR}\left(\mathbf{y}^{*(t)} \mid \delta\right)=\frac{\widehat{m}_{\gamma}^{\mathrm{N}}\left(\mathbf{y}^{*(t-1)} \mid \delta\right)}{\widehat{m}_{\gamma}^{\mathrm{N}}\left(\mathbf{y}^{*(t)} \mid \delta\right)} .
$$

Here we consider comparing the Laplace approximation (LA) to numerical integration (NI), in terms of estimating the above quantity, for the corresponding full models (i.e. models including all covariates under consideration) under the logistic and Poisson simulation scenarios presented in Section 7.2. In Figure C. 1 we present the differences $\log M L R_{L A}-\log M L R_{N I}$ from 1000 MCMC iterations and sample sizes $n=25,50,75,100$. As seen the differences between the two approaches are negligible in all cases.
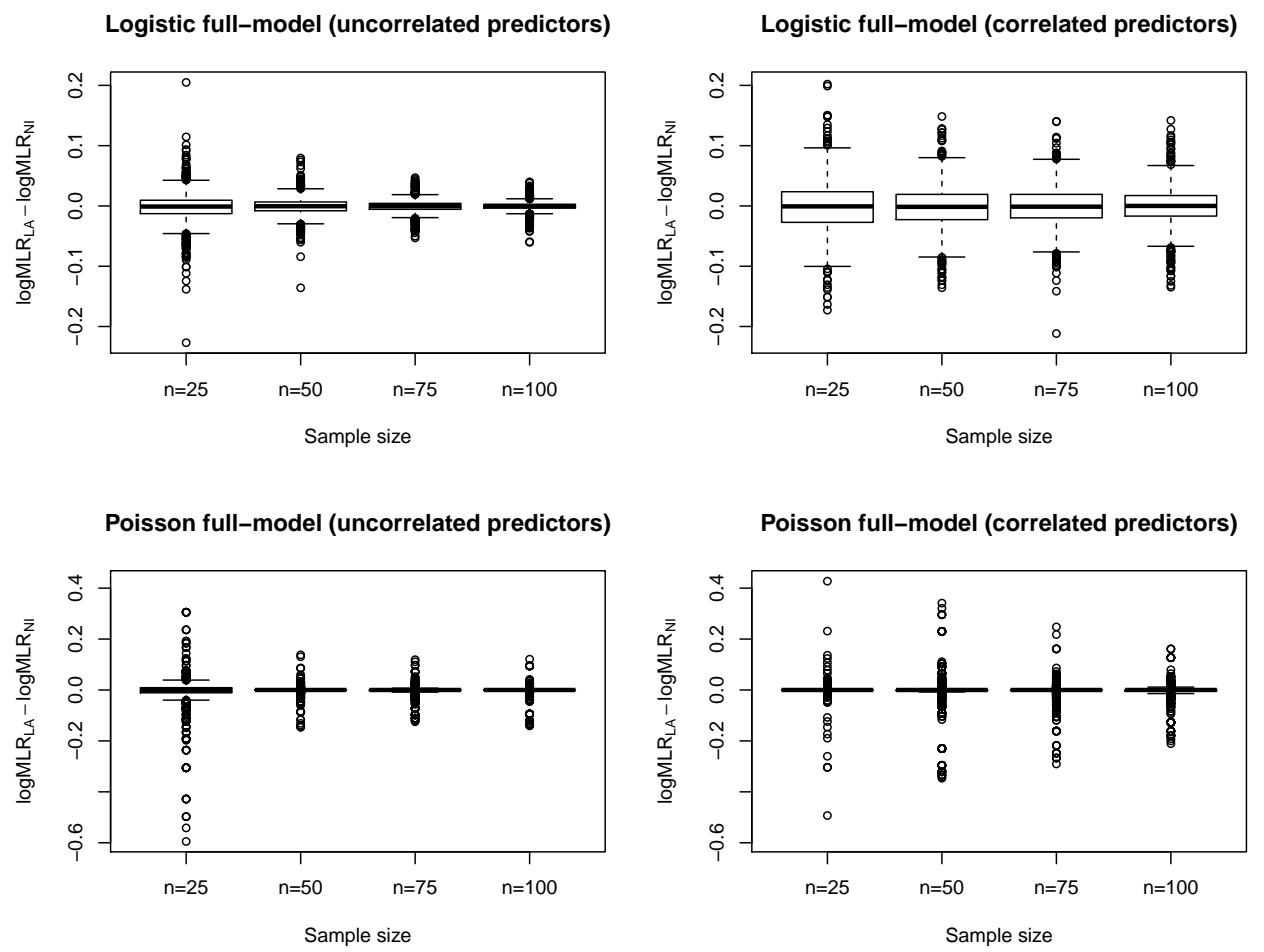

Figure C.1: Log-differences of Laplace approximation MLRs and numerical integration MLRs from 1000 GVS-PEP iterations under different sample sizes. 


\section{Appendix D: Further results from Section 7.2}

In the following, we present boxplots of the inclusion probabilities across the 100 simulated datasets for the logistic simulation scenarios (in Appendix D.1) and for the Poisson simulation scenarios (in Appendix D.2) for the Simulation Study 1 of Section 7.2.

For the logistic case, boxplots of the inclusion probabilities across the 100 simulated datasets are provided in Figure D.1 (independent predictors) and Figure D.2 (correlated predictors) of Appendix D.1. From these figures, we can observe that the DR-PEP based method is quite robust with respect to the choice between fixed versus random $\delta$. Furthermore, within the category of the mixtures of $g$-priors, the MG hyper- $g$ prior has the strongest shrinkage towards zero effect. All methods identify the important effects with similar inclusion probabilities. On the other hand, all methods except for the hyper- $g$ and the hyper- $\delta$ CR-PEP assign very low posterior inclusion probabilities to non-important covariates. The hyper- $g$ and the hyper- $\delta$ CRPEP approach behave in a similar manner, considerably inflating the corresponding inclusion probabilities towards $1 / 2$.

With respect to specific covariate effects, we observe increased uncertainty about the inclusion of $X_{5}$ in the full scenario using independent covariates (for true effect equal to 0.5 corresponding to odds ration equal to 1.65 ). For the correlated scenario, the uncertainty about the important effect is consistently higher in all methods with the highest variability of inclusion probabilities appearing in $X_{2}$ for the medium and full scenarios and in $X_{3}$ for the full scenario.

For the Poisson case, boxplots of the inclusion probabilities across the 100 simulated datasets are provided in Figure D.3 (independent predictors) and Figure D.4 (correlated predictors) of Appendix D.2. Generally, the results are in agreement with the corresponding ones for the logistic case. 
xii

\section{D.1 Posterior inclusion probabilities (logistic model)}
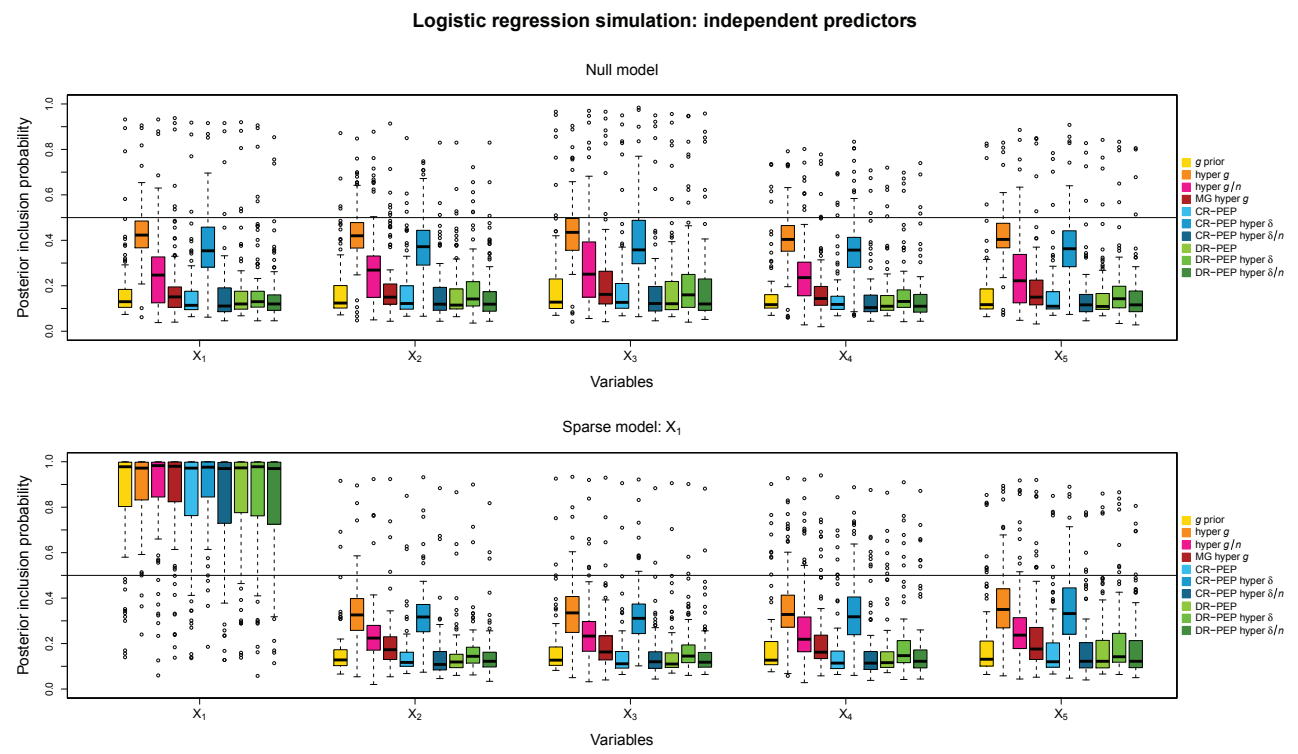

Medium model: $\mathrm{X}_{1}+\mathrm{X}_{2}+\mathrm{X}_{3}$

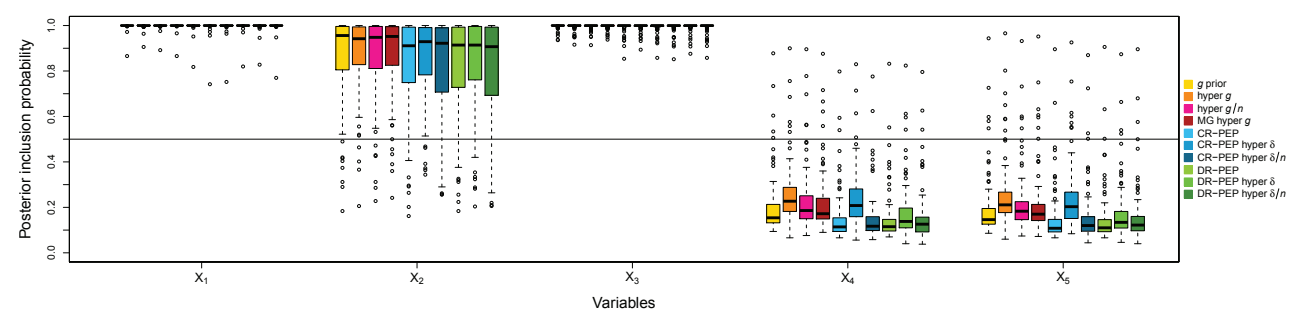

Full model: $\mathrm{X}_{1}+\mathrm{X}_{2}+\mathrm{X}_{3}+\mathrm{X}_{4}+\mathrm{X}_{5}$

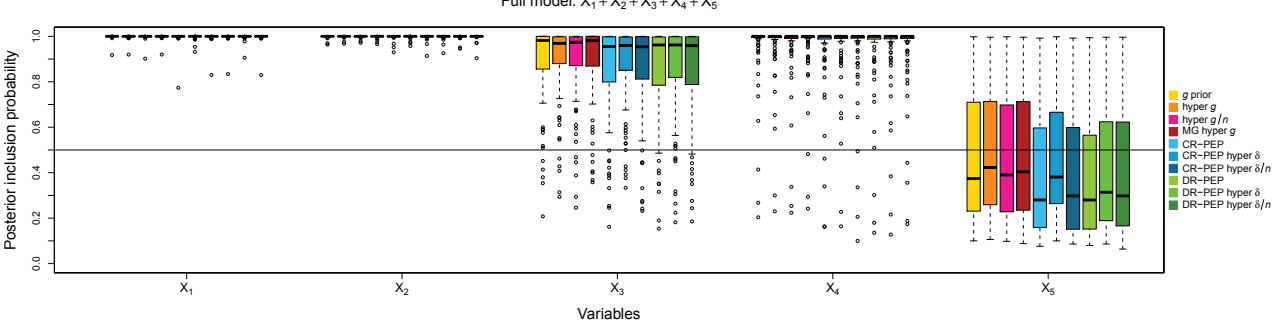

Figure D.1: Posterior inclusion probabilities for Simulation Study 1 from 100 replicated samples of the null, sparse, medium and full logistic regression model scenarios with independent predictors $(r=0)$. 

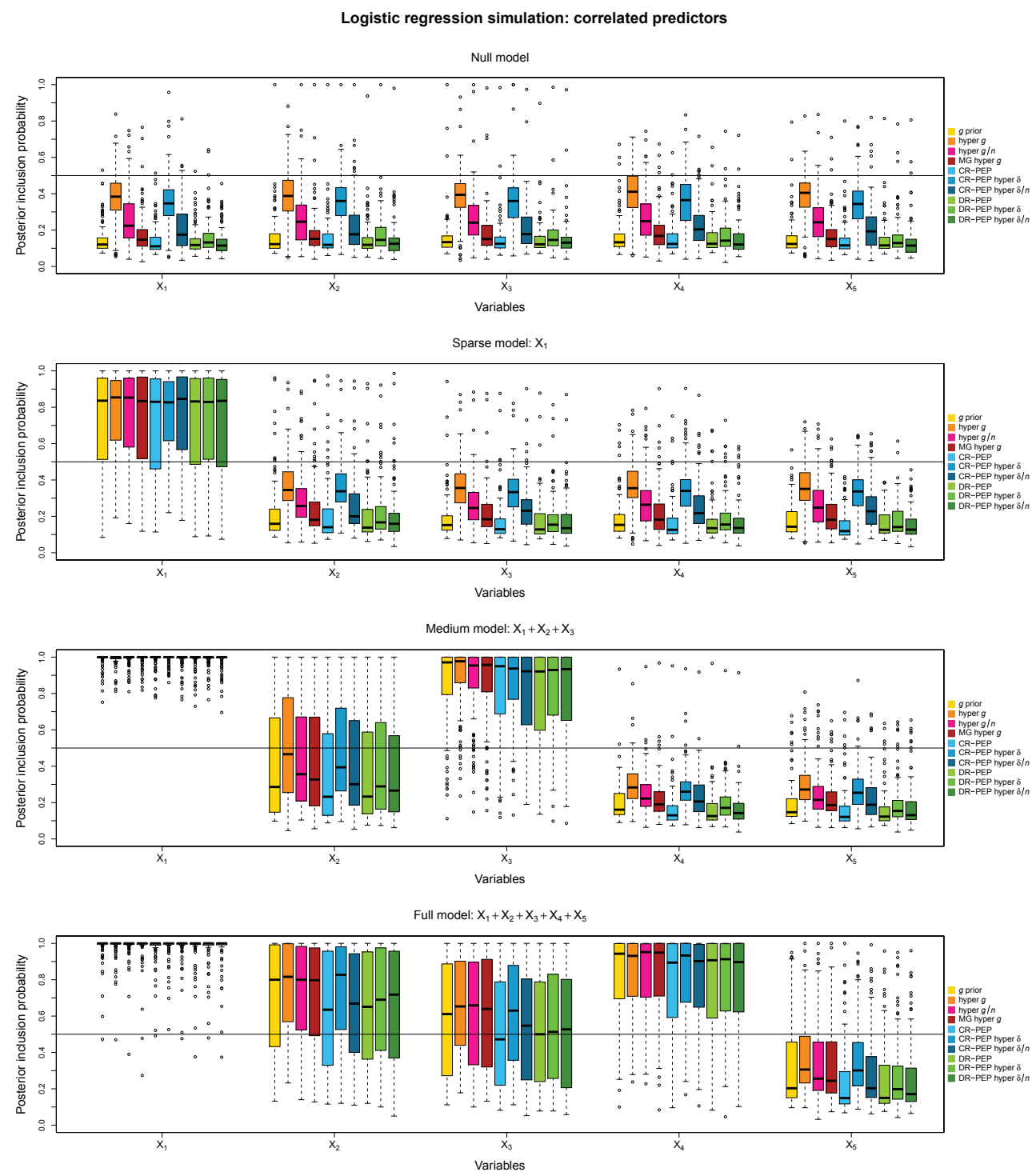

Figure D.2: Posterior inclusion probabilities for Simulation Study 1 from 100 replicated samples of the null, sparse, medium and full logistic regression model scenarios with correlated predictors $(r=0.75)$. 


\section{D.2 Posterior inclusion probabilities (Poisson model)}
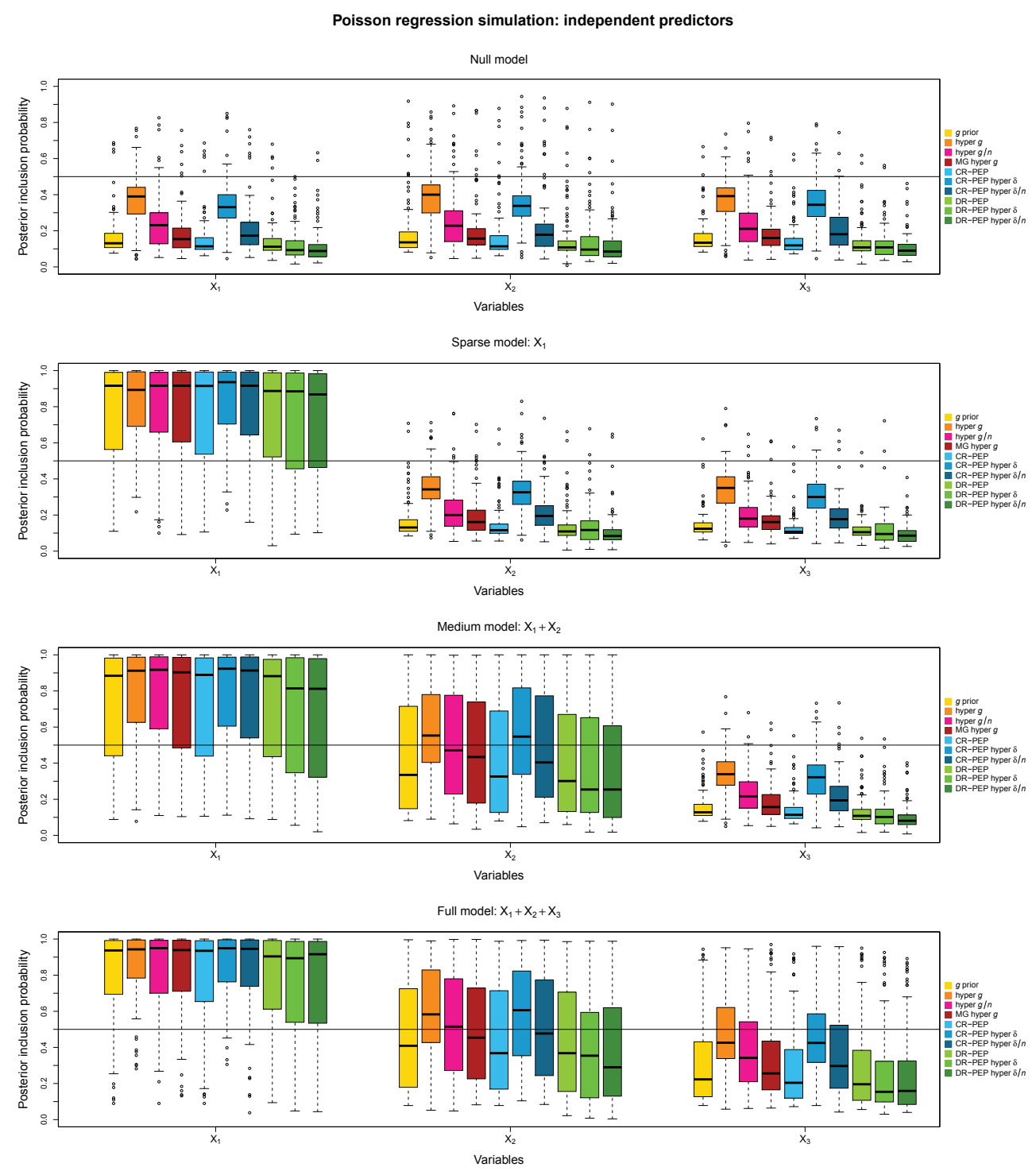

Figure D.3: Posterior inclusion probabilities for Simulation Study 1 from 100 replicated samples of the null, sparse, medium and full Poisson model scenarios with independent predictors $(r=0)$. 

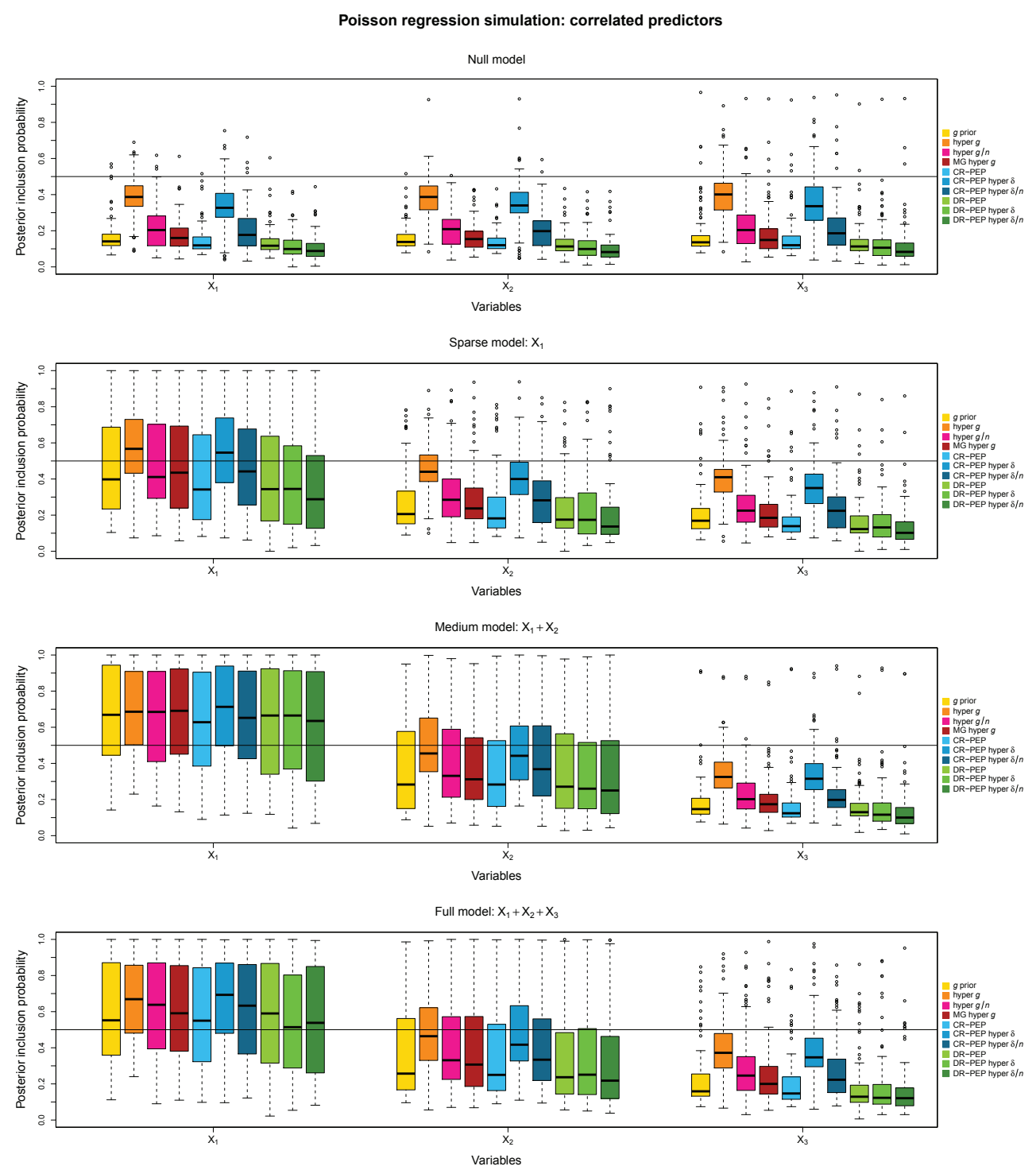

Figure D.4: Posterior inclusion probabilities for Simulation Study 1 from 100 replicated samples of the null, sparse, medium and full Poisson model scenarios with correlated predictors $(r=0.75)$. 


\section{Appendix E: Further results from Section 7.4}

\section{E.1 Posterior inclusion probabilities}

The uncertainty of the estimated posterior inclusion probabilities, for the standard methods considered in the previous examples, is depicted in Figure E.1, where we present the corresponding boxplots produced by splitting the posterior samples into 40 batches of size 1000 . Figure E.1 suggests that stochasticity in $g$ and $\delta$ mainly affects the posterior inclusion probabilities of the "uncertain" covariates $X_{3}, X_{4}$ and $X_{7}$. For these covariates, the extra prior uncertainty induces higher posterior variability, as expected, and consequently larger Monte Carlo errors. Apart from this, we generally observe the same patterns of evidence leading to the conclusions discussed in Sections 7.2 and 7.3.

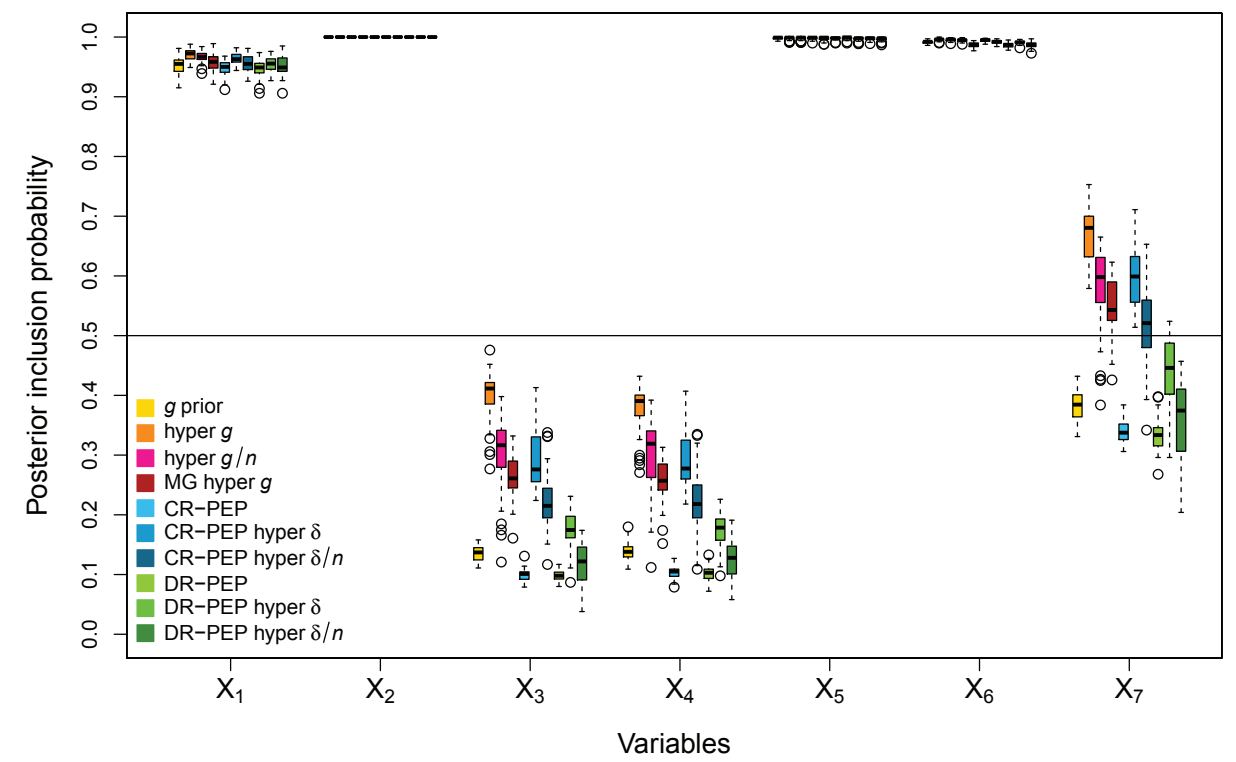

Figure E.1: Boxplots of batched estimates of the posterior inclusion probabilities for the seven predictors in the Pima Indians data set based on 40 batches of size 1000. 


\section{E.2 Convergence plots}

Figure E.2 depicts the convergence and the estimated posterior distribution of the shrinkage parameter $\delta /(1+\delta)$ under the four PEP hyper-prior approaches. The posterior histograms are indicative of the behavior of the shrinkage parameter. Comparison between the hyper- $\delta$ (Figure E.2a) and the hyper- $\delta / n$ (Figure E.2b) approaches shows that the posterior distribution of the shrinkage parameter under the latter priors is more concentrated to values close to one, thus, resulting to a stronger shrinkage effect. Also, the histograms in Figures E.2a and E.2b indicate that the posterior distributions of the shrinkage parameter under DR-PEP are more concentrated to one in comparison to the corresponding posteriors under CR-PEP. Note that the shrinkage under the fixed- $\delta$ approaches is constant, equal to 0.998 , which leads to considerably lower posterior inclusion probabilities as seen in Table 6 in the main paper and Figure E.1.

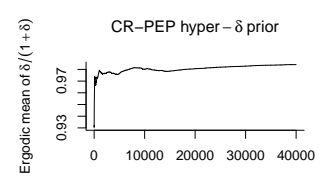

MCMC iterations

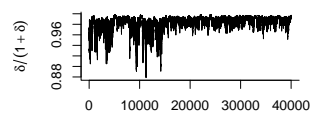

MCMC iterations
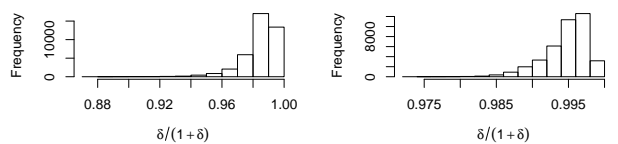

(a) Hyper- $\delta$ Priors

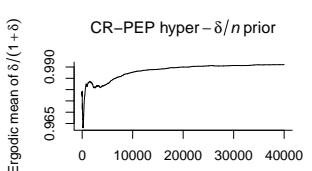

MCMC iterations

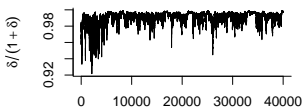

MCMC iterations
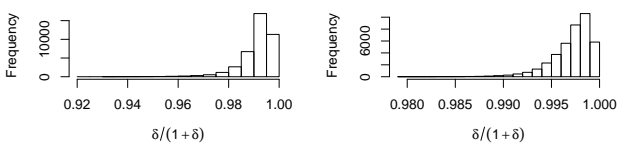

(b) Hyper- $\delta / n$ Priors

Figure E.2: Ergodic mean plots, time-series plots and histograms of the shrinkage factor $\delta /(1+$ $\delta$ ) for the hyper- $\delta$ and hyper- $\delta / n$ PEP priors based on 40000 draws. 